\title{
The bottleneck of CNO burning and the age of Globular Clusters
}

\author{
G. Imbriani ${ }^{1,2,3}$, H. Costantini ${ }^{4}$, A. Formicola ${ }^{5,6}$, D. Bemmerer ${ }^{7}$, R. Bonetti ${ }^{8}$, C. Broggini ${ }^{9}$, P. Corvisiero ${ }^{4}$, J. Cruz ${ }^{10}$, \\ Z. Fülöp ${ }^{11}$, G. Gervino ${ }^{12}$, A. Guglielmetti ${ }^{8}$, C. Gustavino ${ }^{6}$, G. Gyürky ${ }^{11}$, A. P. Jesus ${ }^{10}$, M. Junker ${ }^{6}$, A. Lemut ${ }^{4}$, \\ R. Menegazzo ${ }^{9}$, P. Prati ${ }^{4}$, V. Roca ${ }^{2,3}$, C. Rolfs ${ }^{5}$, M. Romano ${ }^{2,3}$, C. Rossi Alvarez ${ }^{9}$, F. Schümann ${ }^{5}$, E. Somorjai ${ }^{11}$,
} O. Straniero ${ }^{1,2}$, F. Strieder ${ }^{5}$, F. Terrasi ${ }^{2}, 13$, H. P. Trautvetter ${ }^{5}$, A. Vomiero ${ }^{14}$, and S. Zavatarelli ${ }^{4}$

1 INAF-Osservatorio Astronomico di Collurania, Italy

2 INFN-sezione di Napoli, Complesso Universitario di Monte Sant'Angelo, Via Cintia, ed. G80126 Napoli, Italy

${ }^{3}$ Dipartimento di Scienze Fisiche, Universitá Federico II, Complesso Universitario di Monte Sant’Angelo, Via Cintia, ed. G80126 Napoli, Italy

4 Universitá di Genova, Dipartimento di Fisica and INFN, Genova, Italy

5 Ruhr Universität Bochum, Bochum, Germany

${ }^{6}$ INFN Laboratori Nazionali del Gran Sasso, Assergi, Italy

7 Institut für Atomare Physik und Fachdidaktik, Technische Universität Berlin

8 Universitá di Milano, Istituto di Fisica Generale Applicata and INFN, Milano, Italy

9 INFN-sezione di Padova, Italy

${ }^{10}$ Centro de Fisica Nuclear da Universidade de Lisboa, Lisboa, Portugal

11 Atomki, Debrecen, Hungary

12 Universitá di Torino, Dipartimento di Fisica Sperimentale and INFN, Torino, Italy

13 Seconda Universitá di Napoli, Dipartimento di Scienze Ambientali, Caserta, and INFN, Napoli, Italy

14 Universitá di Padova, Dipartimento di Fisica, Padova and INFN Legnaro, Italy

Received 6 January 2004 / Accepted 1 March 2004

\begin{abstract}
The transition between the Main Sequence and the Red Giant Branch in low mass stars is powered by the onset of CNO burning, whose bottleneck is ${ }^{14} \mathrm{~N}(\mathrm{p}, \gamma){ }^{15} \mathrm{O}$. The LUNA collaboration has recently improved the low energy measurements of the cross section of this key reaction. We analyse the impact of the revised reaction rate on the estimate of the Globular Cluster ages, as derived from the turnoff luminosity. We found that the age of the oldest Globular Clusters should be increased by about $0.7-1$ Gyr with respect to the current estimates.
\end{abstract}

Key words. stars: evolution - stars: population II - nuclear reactions, nucleosynthesis, abundances Galaxy: globular clusters: general - Galaxy: formation - cosmology: distance scale

\section{Introduction}

Globular Clusters (GCs) represent the oldest resolved stellar populations. Their age practically coincides with the time elapsed since the epoch of the formation of the first stars in the Universe and provides an independent check of the reliability of standard (and non-standard) cosmological models. Moreover, the age spread in the GC system is a primary indicator of the time scale of the halo formation. Among the various methods to date stellar Clusters, the most reliable and widely adopted is that based on the measurement of the luminosity of the turnoff (i.e. the bluest point on the main sequence). This dating technique requires the knowledge of the Cluster distance, the light extinction along the line of sight and the chemical composition (see Gratton et al. 2003 for an exhaustive analysis of the present state of the art). In addition, a reliable theoretical calibration of the turnoff luminosity-age

Send offprint requests to: G. Imbriani, e-mail: imbriani@na.infn.it relation (TOL-A) is needed. This relies on our knowledge of the physical processes of energy generation (e.g. nuclear reactions) and transport (e.g. opacity) taking place in H-burning low mass stars. An adequate description of the thermodynamics of stellar matter is also required. Finally we have to consider any mechanism able to modify the internal chemical stratification (once again nuclear reactions, convective mixing, rotationinduced mixing, microscopic diffusion or levitation induced by radiation pressure). Chaboyer et al. (1996) discuss the influence of various theoretical uncertainties on the calibration of the turnoff luminosity-age relation and conclude that the total uncertainty due to the theory may be confined within $0.5 \mathrm{Gyr}$.

This paper is devoted to the evaluation of the impact on the theoretical calibration of the Globular Cluster ages of the improved determination of the rate of the key reaction ${ }^{14} \mathrm{~N}(\mathrm{p}, \gamma){ }^{15} \mathrm{O}$, as obtained by the LUNA collaboration (Formicola et al. 2003b). Since thermonuclear reactions are responsible for chemical modifications occurring in stellar interiors, and supply most of the energy irradiated from the 
stellar surface, the estimated stellar lifetime depends on accurate measurements of their rates. In the last few years, much effort has been spent in improving these measurements at energies as close as possible to the Gamow peak, namely the relevant energies at which nuclear reactions take place in stars. This is a mandatory requirement for the calibration of stellar ages.

The main sequence stars presently observed in Globular Clusters have masses smaller than that of the Sun. As in the Sun, these low mass stars burn $\mathrm{H}$ in the center, mainly through the pp chain. However, towards the end of their life, when the central hydrogen mass fraction becomes smaller than about 0.1 , the nuclear energy released by the $\mathrm{H}$ burning becomes insufficient and the stellar core must contract to extract some energy from its own gravitational field. Then, the central temperature (and the density) increases and the $\mathrm{H}$ burning switches from the pp chain to the more efficient CNO cycle. Thus, the departure from the Main Sequence is powered by the CNO cycle, whose bottleneck is the ${ }^{14} \mathrm{~N}(\mathrm{p}, \gamma){ }^{15} \mathrm{O}$ reaction. The luminosity of the turnoff depends on the rate of this key reaction: the larger the rate, the fainter the turnoff. In contrast, the total lifetime is only marginally affected by a change in the CNO, because it is mainly determined by the rate of the ${ }^{1} \mathrm{H}\left(\mathrm{p}, \mathrm{e}^{+}, v_{\mathrm{e}}\right)^{2} \mathrm{H}$. As a consequence, an increase of the $\mathrm{CNO}$ rate would imply fainter turnoff points for a given age, or younger ages for a given turnoff luminosity (see also Chaboyer et al. 1998). Note that an equivalent effect may be caused by the enhancement of the CNO abundances (Rood 1981; Salaris et al. 1993).

In the next section we recall the new measurements of the stellar cross section of the ${ }^{14} \mathrm{~N}(\mathrm{p}, \gamma){ }^{15} \mathrm{O}$ reaction. Then, in Sect. 3 we present the revised turnoff luminosity-age relation (TOL-A). We show that this revision leads to systematically larger estimates of the age of the Globular Clusters. Implications for cosmology are briefly discussed in the conclusive section.

\section{The updated ${ }^{14} \mathrm{~N}(\mathrm{p}, \gamma){ }^{15} \mathrm{O}$ reaction rate}

The minimum energy explored in direct on-line $\gamma$ measurements before $\mathrm{LUNA}^{1}$ was $\sim 240 \mathrm{keV}$, which is well above the range of interest for stellar CNO burning $(\sim 20-80 \mathrm{keV})$. Therefore, the reaction rate used in stellar model computations is largely extrapolated, in a region where the resonant structure of the ${ }^{15} \mathrm{O}$ compound nucleus is particularly complex. The rates reported by the popular compilations (Caughlan \& Fowler 1988, CF88; and Angulo et al. 1999, NACRE), which are based on the cross section measurements obtained by Schröder et al. (1987) are very similar. In particular, the astrophysical factor at zero energy is $S(0)=3.2 \pm 0.8 \mathrm{keV} \mathrm{b}$ (NACRE). The main contributions to $S(0)$ come from the transitions to the groundstate in ${ }^{15} \mathrm{O}$ and to the subthreshold state at $E_{\mathrm{cm}}=-504 \mathrm{keV}$. It is the existence of this subthreshold resonance that makes the extrapolation very uncertain. Recently Angulo \& Descouvement (2001), reanalyzing Schröder's experimental data by means of a R-matrix

${ }^{1}$ LUNA is an acronym of the Laboratory for Underground Nuclear Astrophysics, operating at the LNGS of Assergi, Italy.

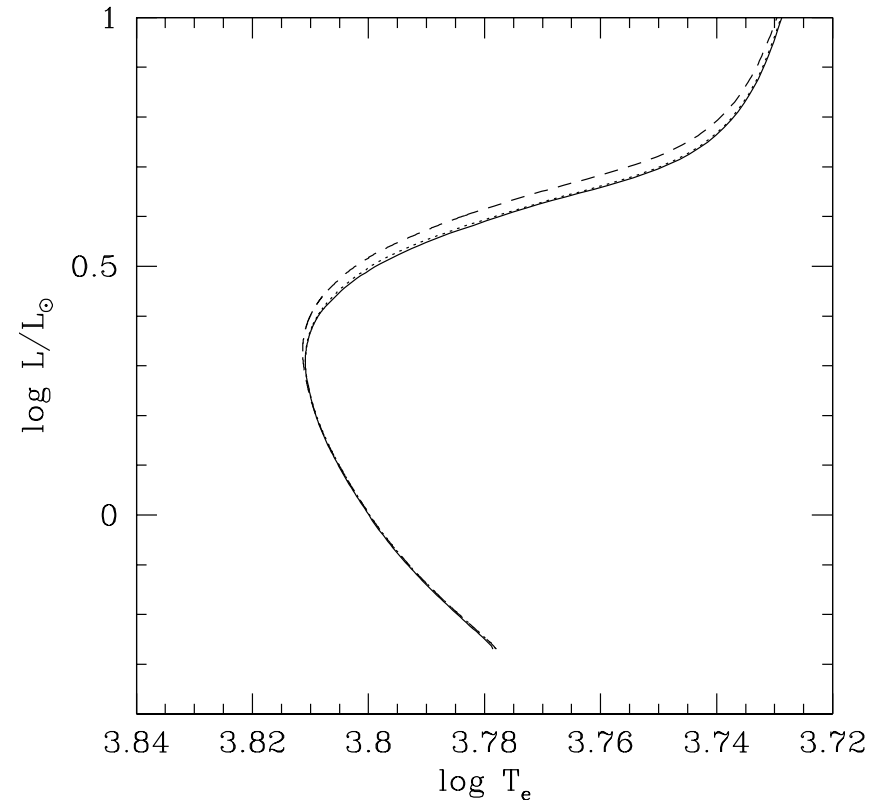

Fig. 1. Comparison between evolutionary sequences obtained with different rates of ${ }^{14} \mathrm{~N}(\mathrm{p}, \gamma){ }^{15} \mathrm{O}$ : NACRE (dotted line), CF88 (solid line) and LUNA (dashed line). The stellar mass is $0.8 M_{\odot}$ and the metallicity is $Z=0.0003$.

model, report a significant lower $S(0)$, namely $1.77 \pm 0.20 \mathrm{keV} \mathrm{b}$ (see also Nelson et al. 2003). The main discrepancy concerns the contribution of the captures to the ${ }^{15} \mathrm{O}$ groundstate, which has been found to be 19 times smaller than the value quoted by Schröder and adopted by NACRE and CF88. We emphasize that the large discrepancy among different analyses based on the same data set is a clear demonstration of the inadequacy of the low energy extrapolation for this reaction.

The LUNA collaboration has significantly improved the low energy measurements of this reaction rate (Formicola et al. 2003b). We used a $400 \mathrm{keV}$ facility (Formicola et al. 2003a), which is particularly well suited when reaction $\gamma$-ray lines up to $\simeq 7.5 \mathrm{MeV}$ have to be measured with very low intensities. Cosmic background is strongly suppressed by the mountain shielding and low intrinsic activity detectors are employed. The explored energy window ranges from $390 \mathrm{keV}$ down to $135 \mathrm{keV}$, i.e., significantly closer to the astrophysical relevant energy than any previous experiment. The fit of the new data by means of a R-matrix model leads to $S(0)=1.7 \pm 0.1$ (stat) \pm 0.2 (sys) $\mathrm{keV}$ b. In the following we use this result to revise the calibration of the turnoff luminosity-age relation.

\section{Globular Clusters ages}

New stellar models have been computed with the same code described in Straniero et al. (1997), but updating the rate of ${ }^{14} \mathrm{~N}(\mathrm{p}, \gamma){ }^{15} \mathrm{O}$. We recall that this code includes an improved equation of state (which properly takes into account the degree of degeneracy of the electrons and the electrostatic interactions), the most recent compilation of opacity for stellar interiors (Iglesias et al. 1992; Alexander \& Ferguson 1994) and microscopic diffusion (Thoul et al. 1994). Figure 1 shows an example of the evolutionary track obtained by adopting 


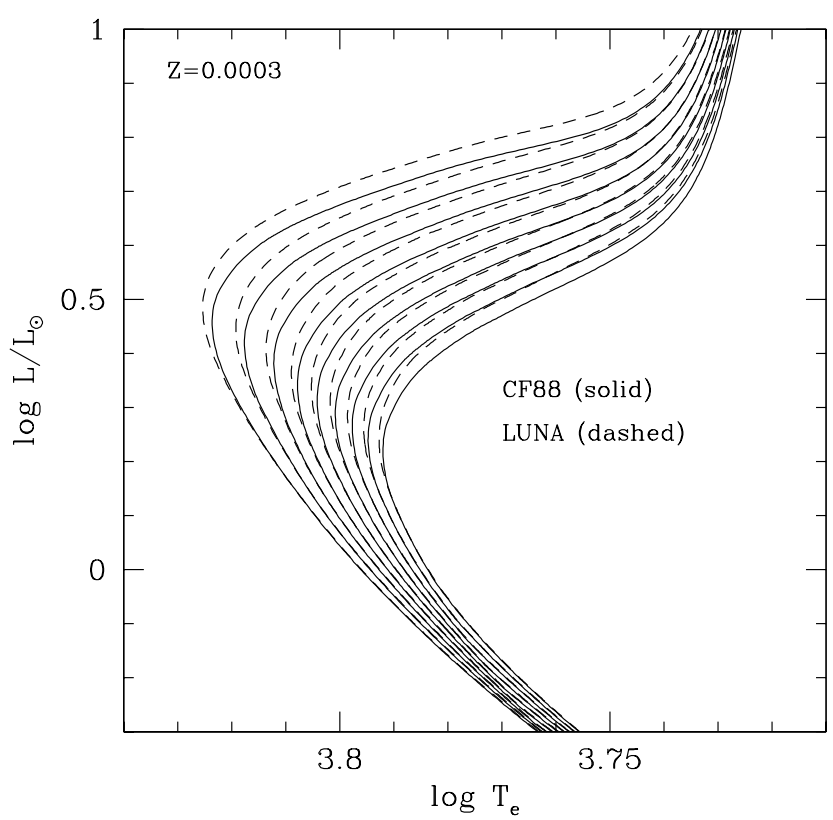

Fig. 2. Isochrones for Globular Clusters obtained with different rates of the ${ }^{14} \mathrm{~N}(\mathrm{p}, \gamma){ }^{15} \mathrm{O}$ reaction: CF88 (solid) and LUNA (dashed). The brightest isochrone (of each set) is the youngest (10 Gyr), while the fainter is the oldest (18 Gyr). The age step between two adjacent isochrones is $1 \mathrm{Gyr}$. The metallicity is $Z=0.0003$ (or $[\mathrm{M} / \mathrm{H}]=-1.82)$.

different rates for the ${ }^{14} \mathrm{~N}(\mathrm{p}, \gamma){ }^{15} \mathrm{O}$ reaction. The tracks obtained with the CF88 rate practically coincide with the one obtained with the NACRE rate. In contrast, the turnoff and the subgiant branch of the sequences obtained by adopting the new rate are substantially brighter.

Isochrones have been computed for two sets of stellar models, the first based on the old CF88 rate and the second based on the revised LUNA rate. We have explored the whole range of chemical composition covered by the galactic GC system. In particular, the mass fraction of metals (the metallicity) has been varied between $Z=0.0001$ and $Z=0.006$, which corresponds to $[\mathrm{M} / \mathrm{H}]=-2.3$ and $[\mathrm{M} / \mathrm{H}]=-0.5^{2}$. Some examples of the comparison between old and new isochrones are shown in Figs. 2 and 3. As expected, the lower rate of ${ }^{14} \mathrm{~N}(\mathrm{p}, \gamma){ }^{15} \mathrm{O}$ leads to brighter and bluer turnoff points (for a given age). When a given turnoff luminosity is considered, the revised isochrones imply systematically older ages, namely between 0.7 and $1 \mathrm{Gyr}$.

To compare our isochrones to the available photometric studies of globular cluster stars, we have transformed luminosities and effective temperatures into magnitudes and colors by means of model atmospheres provided by Castelli et al. (1997). The accuracy of the new isochrones in reproducing the morphology of the observed color-magnitude diagrams, has been checked by selecting two clusters which are representative of the oldest component of the galactic halo. The first test is illustrated in Fig. 4. Isochrones for $Z=0.0003([\mathrm{M} / \mathrm{H}]=-1.8)$ and age 13,14 and 15 Gyr are superimposed on the color magnitude diagram of NGC 6397. A similar test, but for NGC 5904 (M 5),

\footnotetext{
${ }^{2}$ Standard spectroscopic notation.
}

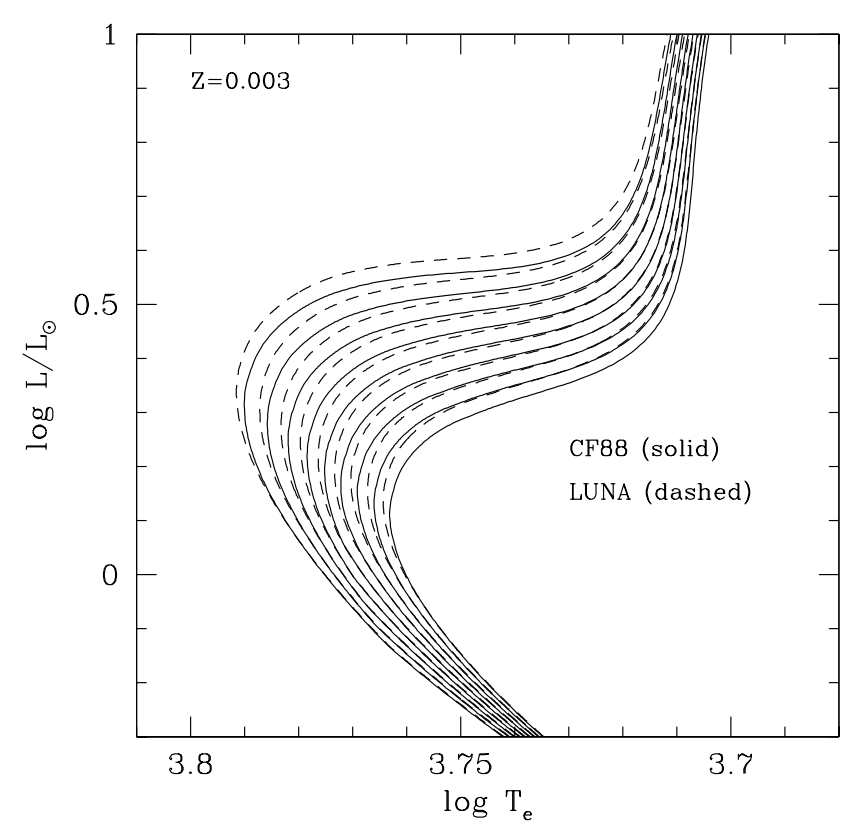

Fig. 3. Isochrones for Globular Clusters obtained with different rates of the ${ }^{14} \mathrm{~N}(\mathrm{p}, \gamma){ }^{15} \mathrm{O}$ reaction: CF88 (solid) and LUNA (dashed). The brightest isochrone (of each set) is the youngest (10 Gyr), while the fainter is the oldest $(18 \mathrm{Gyr})$. The age step between two adjacent isochrones is $1 \mathrm{Gyr}$. The metallicity is $Z=0.003([\mathrm{M} / \mathrm{H}]=-0.52)$ in the right panel.

is reported in Fig. 5, where the isochrones have $Z=0.001$ $([\mathrm{M} / \mathrm{H}]=-1.3)$. Photometric data are from the ground-based database published by Rosenberg et al. (2000). In both cases, the new isochrones match the overall color-magnitude diagram well at $14 \mathrm{Gyr}$, with a bona fide uncertainty of \pm 1 Gyr. Similar results were obtained by Straniero et al. (1997) with the old (CF88) isochrones, but in that case the best reproduction of the observed diagrams required $13 \mathrm{Gyr}$ (see their Fig. 11).

The following relation for the Globular Cluster age ( $t_{9}$ in Gyr), as a function of the $V$ magnitude of the turnoff point $\left(M_{V}^{\mathrm{TO}}\right)$ and the metallicity $([\mathrm{M} / \mathrm{H}])$, has been derived.

$$
\begin{aligned}
\log t_{9}= & -1.0146-0.2731[\mathrm{M} / \mathrm{H}]+0.03032[\mathrm{M} / \mathrm{H}] M_{V}^{\mathrm{TO}} \\
& -0.00058\left([\mathrm{M} / \mathrm{H}] M_{V}^{\mathrm{TO}}\right)^{2}+0.4801 M_{V}^{\mathrm{TO}}
\end{aligned}
$$

The standard deviation of the estimated age is $\Delta \log t_{9}=0.005$. This relation can be used for ages ranging between 10 and $18 \mathrm{Gyr}$ and $[\mathrm{M} / \mathrm{H}]$ between -2.3 and -0.5 .

\section{Implications for cosmology}

The recent developments of accurate measurements of the fundamental cosmological parameters allow us to derive a very precise age of the Universe: $t_{0}=13.7 \pm 0.2 \mathrm{Gyr}$ (Spergel et al. 2003). This result has been obtained in the framework of a $\Lambda \mathrm{CDM}$ model, and it is based on the measures of three fundamental parameters: $H_{0}$, whose best determination has been obtained by the Key HST Project (Freedman et al. 2001), $\Omega$, measured by WMAP (Spergel et al. 2003), and the ratio $\Omega_{M} / \Omega_{\Lambda}$, constrained by the observation of type Ia supernovae in high redshift galaxies (Perlmutter et al. 1999; Smith et al. 1998). 


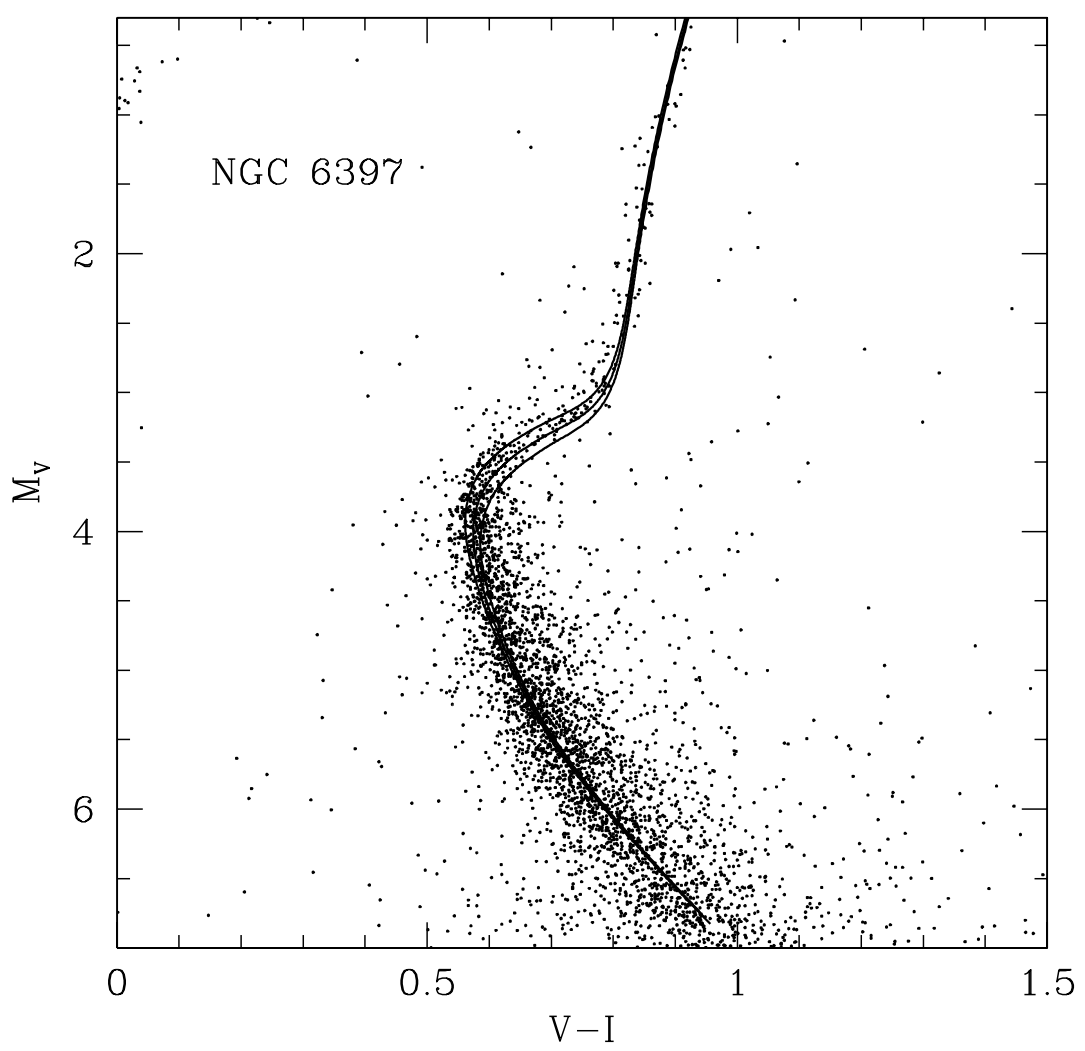

Fig. 4. Test of the CMD of the metal-poor cluster NGC 6397. The new isochrones with 13,14 and 15 Gyr are reported. Their metallicity is $Z=0.0003([\mathrm{M} / \mathrm{H}]=-1.8)$. We adopt $(m-M)_{V}=12.58$ and $E(B-V)=0.18$. The data are from Rosenberg et al. (2000).

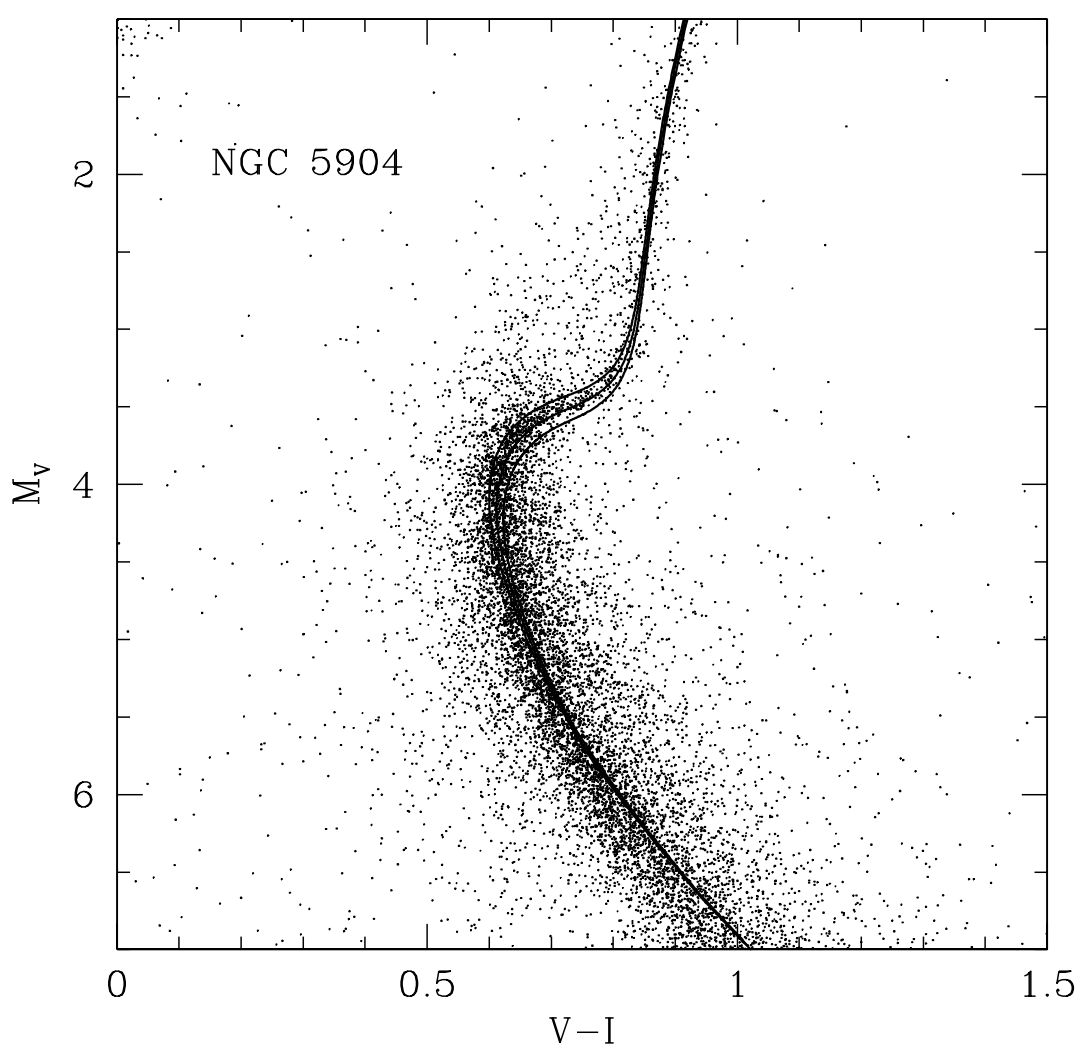

Fig. 5. Test of the CMD of the intermediate metallicity cluster NGC 5904. The new isochrones with 13,14 and 15 Gyr are presented. Their metallicity is $Z=0.001([\mathrm{M} / \mathrm{H}]=-1)$. We adopt $(m-M)_{V}=14.41$ and $E(B-V)=0.02$. The data are from Rosenberg et al. (2000). 
The galaxy clustering shape measurements also constrain $\Omega_{M}$ (Percival et al. 2001). It is obvious that any systematic uncertainty affecting just one of these experiments would imply a revision of this estimate of the age of the Universe. For example, it has been argued that the light curve of an SNe Ia might depend on the chemical composition and/or the mass of the progenitor star. In this case, the commonly assumed similarity between nearby and high redshift SNe Ia, in spite of the different stellar populations of their host Galaxies, could have induced a systematic error in the evaluation of $\Omega_{M} / \Omega_{\Lambda}$ (see e.g., Domínguez et al. 2001).

In this context, an independent determination of the age of the Universe, may (or may not) confirm the standard cosmological model that emerges from experimental cosmology. At present, the most reliable dating technique is the one based on the TOL-A relation for the oldest stellar systems of the Milky Way, the Globular Clusters. The standard cosmological model also predicts that the $\mathrm{H}$ reionization, which should coincide with the epoch of the first star formation, occurred between 100 and 400 Myr after the Big Bang (95\% CL, Spergel et al. 2003). Such a delay must also be considered.

An exhaustive comparison between stellar and cosmological ages requires a detailed statistical analysis taking into account all sources of errors (experimental and theoretical). This is beyond the purpose of the present paper and will be presented elsewhere. Let us limit our discussion to the expected implication of the revised ${ }^{14} \mathrm{~N}(\mathrm{p}, \gamma){ }^{15} \mathrm{O}$ reaction rate. We have shown that the revised ages of the Globular Clusters are older, about 0.7-1 Gyr, than those previously claimed. Note that, in the framework of the $\Lambda \mathrm{CDM}$ model, an equivalent increase of $t_{0}$ might be obtained by reducing $H_{0}(\sim 5 \%)$ or $\Omega_{M}(\sim 8 \%)$. These variations are, in any case, within the experimental errors.

Gratton et al. (2003), by means of the TOL-A relation derived from models taking into account the effect of microscopic diffusion, but computed adopting the old ${ }^{14} \mathrm{~N}(\mathrm{p}, \gamma){ }^{15} \mathrm{O}$, conclude that the age of the oldest Galactic Clusters is $13.4 \mathrm{Gyr}$ ( \pm 0.8 random, \pm 0.6 systematic). When the age increment implied by the revision of the ${ }^{14} \mathrm{~N}(\mathrm{p}, \gamma){ }^{15} \mathrm{O}$ is just added, the best fit to the age of the oldest GCs should increase to above 14 Gyr. This revised lower limit of the age of the Universe strengthens the need of a positive cosmological constant. In the case of a flat Universe $(\Omega=1)$ and assuming $H_{0}=72 \mathrm{~km} \mathrm{~s}^{-1} \mathrm{Mpc}^{-1}$ (Freedman et al. 2001), a Universe older than $14 \mathrm{Gyr}$ would imply $\Omega_{M}<0.22$ or, adopting an uncertainty of $1.4 \mathrm{Gyr}$ in $t_{0}$ (Gratton et al. 2003), $\Omega_{M}<0.35$. Note that this upper limit for the matter density is independent of the $\mathrm{SNe}$ Ia observations. Alternatively, by coupling our result with that of the high redshift SNe Ia, we may relax the assumption for the geometry of the Universe to derive a stringent constraint for the Hubble constant. Indeed, taking $H_{0} t_{0}=0.96 \pm 0.04$ (Tonry et al. 2003), the present lower limit for $t_{0}$ would imply $H_{0}<67 \mathrm{~km} \mathrm{~s}^{-1} \mathrm{Mpc}^{-1}$ (or $H_{0}<74$ within $1 \sigma$ in $t_{0}$ ), in good agreement with $72 \pm 8$ obtained by the Key HST Project (Freedman et al. 2001).

Acknowledgements. We are indebted to the referee (A. Weiss), who found a mistake in the first version of the paper. This work has been partially supported by the Italian grant COFIN 2001 and by FEDER (POCTI/FNU/41097/2001).

\section{References}

Alexander, D. R., \& Ferguson, J. W. 1994, ApJ, 437, 879

Angulo, C., Arnould, M., Rayet, M., et al. 1999, Nucl. Phys. A, 656, 3 (NACRE)

Angulo, C., \& Descouvemont, P. 2001, Nucl. Phys. A, 690, 755

Castelli, F., Gratton, R. G., \& Kurucz, R. L., et al. 1997, A\&A, 318, 841

Caughlan, G. R., \& Fowler, W. A. 1988, A.D.N.D.T., 40, 283 (CF88)

Chaboyer, B., Demarque, P., Kernan P. J., \& Kraus, L. M. 1996, Science, 271, 975

Chaboyer, B., Demarque, P., Kernan, P. J., \& Kraus, L. M. 1998, ApJ, 494, 96

Domínguez, I., Höflich, P., \& Straniero, O. 2001, ApJ, 557, 279

Formicola, A., Imbriani, G., Junker, M., et al. 2003a, Nucl. Instr. Meth. A, 507, 609

Formicola, A., Imbriani, G., Costantini, H., et al. 2003b, Phys. Lett. B, submitted (LUNA) [nucl-ex/0312015]

Freedman, W. L., Madore, B. F., Gibson, B. K., et al. 2001, ApJ, 553, 47

Gratton, R. G., Bragaglia, A., Carretta, E., et al. 2003, A\&A, 408, 529

Iglesias, C. A., Rogers, F. J., \& Wilson, B. G. 1992, ApJ, 397, 717 (OPAL)

Nelson, S. O., Ahmed, M. W., Perdue, B. A., et al. 2003, Phys. Rev. C, 68, 065804

Percival, W. J., Baugh, C. M., Bland-Hawthorn, J., et al. 2001, MNRAS, 327, 1297

Perlmutter, S., Aldering, G., Goldhaber, G., et al. 1999, ApJ, 517, 565

Rood, R. T. 1981, in Physical Process in Red Giants, ed. I. Iben Jr., \& A. Renzini (Dordrecht: Reidel), 51

Rosenberg, A., Piotto, G., Saviane, I., \& Aparicio, A. 200, A\&A, 144, 5

Schröder, U., Becker, H. W., Bogaert, G., et al. 1987, Nucl. Phys. A, 467,240

Salaris, M., Chieffi, A., \& Straniero, O. 1993, ApJ, 414, 580

Schmidt, B. P., Suntzeff, N. B., Phillips, M. M., et al. 1998, ApJ, 507, 46

Spergel, D. N., Verde, L., Peiris, H. V., et al. 2003, ApJS, 148, $175 \mathrm{~S}$

Straniero, O., Chieffi, A., \& Limongi, M. 1997, ApJS, 490, 425

Thoul, A. A., Bahcall, J. N., \& Loub, A. 1994, ApJ, 421, 828

Tonry, J. L., Schmidt, B. P., Barris, B., et al. 2003, ApJ, 594, 1 